\title{
Tangence
}

\section{Au jour le jour}

\section{Catherine Maubon}

Numéro 42, décembre 1993

Le récit de soi

URI : https://id.erudit.org/iderudit/025787ar

DOI : https://doi.org/10.7202/025787ar

Aller au sommaire du numéro

Éditeur(s)

Tangence

ISSN

0226-9554 (imprimé)

1710-0305 (numérique)

Découvrir la revue

Citer cet article

Maubon, C. (1993). Au jour le jour. Tangence, (42), 27-48.

https://doi.org/10.7202/025787ar d'utilisation que vous pouvez consulter en ligne.

https://apropos.erudit.org/fr/usagers/politique-dutilisation/ 


\section{Au jour le jour ${ }^{1}$ \\ Catherine Maubon}

Au coeur de l'hiver 1932, dans une chambre d'hôtel de province, prend forme, sous la plume d'un jeune historien en proie à un malaise dont le sens lui échappe, l'un des projets d'écriture les plus précis de la littérature de notre temps:

Le mieux serait d'écrire les événements au jour le jour. Tenir un journal pour y voir clair. Ne pas laisser échapper les nuances, les petits faits, même s'ils n'ont l'air de rien, et surtout les classer. Il faut dire comment je vois cette table, la rue, les gens, mon paquet de tabac, puisque c'est cela qui a changé. Il faut déterminer exactement l'étendue et la nature de ce changement. ${ }^{2}$

Un véritable modèle du genre pour un genre - le journal qui aurait plutôt tendance à manquer de modèle. Ou, si l'on préfère, dont aucune des nombreuses et illustres exemplifications qui en ponctuent le développement depuis le début du XIX ${ }^{\mathrm{e}}$ siècle n'a réussi à cerner d'aussi près le statut. Mais comment Antoine Roquentin - c'est de lui qu'il s'agit - a-t-il pu projeter d'emblée et apparemment sans hésitations ce que d'autres ont mis une vie entière à chercher à définir, incapables comme ils l'étaient de réunir, sous un dénominateur commun, les multiples formes que prenait, au fil des ans, un projet qui, le plus souvent, ne se révélait tel que rétrospectivement, au moment où ils en mesuraient les difficultés et les embûches?

Des blancs de plus d'un mois. Parler de moi m'ennuie; un journal est utile dans les évolutions morales conscientes, voulues et difficiles. On veut savoir où l'on en est. Mais ce que je dirais maintenant, ce serait des ressassements sur moi-même. un journal intime est intéressant surtout quand il note l'éveil des idées; ou des sens, lors de la puberté; ou bien enfin lorsqu'on se sent mourir. ${ }^{3}$

1 Je présente ici la première partie de l'Appendice d'un ouvrage en cours de publication: Michel Leiris. Dans les manges de l'autobiographie, Paris, Corti, 1994.

2 Jean-Paul Sartre, La nausée, in Euvres romanesques, Paris, Gallimard, "Bibliothèque de la Pléiade", 1981, p. 5.

3 André Gide, Journal, 1889-1939, Paris, Gallimard, "Bibliothèque de la Pléiaden, 1948 , p. 26. Â ce propos et, plus généralement, sur la poétique du 
Gide tient son journal depuis deux ans quand, le 8 octobre 1891, il se heurte pour la première fois au risque d'enlisement dans sa propre parole qui menace celui qui s'y aventure sans savoir pourquoi ni comment. Un risque auquel saura échapper Roquentin et pour cause. Commencé le 25 janvier, son journal s'arrête le 24 février 1932, au moment où, de dévoilement en dévoilement, il aura en quelque sorte surmonté, au jour le jour, la crise suscitée par la découverte de la contingence. Comme si le journal était ce que certains voudraient qu'il soit, une œuvre progressive, porteuse d'un sens hégémonique. Mais, on le sait, Roquentin n'est pas l'auteur de son journal. La nausêe est un roman qui a trouvé dans l'écriture quotidienne la forme (fictionnelle) la plus appropriée à la résolution (philosophique) de l'énigme existentielle à laquelle Sartre entendait apporter une réponse immédiatement déchiffrable.

Un journal donc comme en écrivent "les petites filles dans un beau cahier neuf" mais aussi les jeunes gens qui savent ou ne savent pas qu'ils seront un jour considérés comme de grands écrivains. Ces journaux, on s'en doute, n'ont pas la compacité de celui de Roquentin. Aucun schéma narratif ni discursif ne préside à leur difficile élaboration. Leurs narrateurs travaillent dans le vide et très souvent il leur arrive de tomber. Certains ne se relèvent plus. D'autres, tel Leiris, continuent et se retrouvent à l'autre bout de leur existence sans avoir résolu quoi que ce soit. Ils ont acumulé des feuillets, rempli des cahiers mais n'ont connu aucune révélation:

L'espoir de trouver ce que je cherche s'est, pour moi, réduit peu à peu à celui de trouver, non pas la chose que je cherche, mais quelle est exactement la chose que je voudrais trouver. Bref, ce qu'aujourd'hui je cherche c'est ce qu'est ce que je cherche (26 août 1969). ${ }^{4}$

Aujourd'bui, c'est-à-dire après quarante sept ans de quête. Bien qu'il alimente le fantasme de la réponse dans la possibilité qui lui appartient en propre de renouveler la question, le journal ne saurait dévoiler un secret qui n'existe que dans l'acte de

Journal, E. Marty, L'écriture du jour - le Journal d'André Gide, Paris, Seuil, 1985.

4 Michel Leiris, Journal, édition établie, présentée et annotée par J. Jamin, Paris, Gallimard, 1992, p. 640. Les citations seront désormais suivies de la seule indication de la date et de la page. 
parole qui en postule la réalité. Son but n'est pas, ainsi que beaucoup le croient, initialement du moins, d'atteindre un hypothétique point d'arrivée mais d'assurer les conditions de son prolongement. De trouver, dans ce prolongement, une légitimation qui, à tout instant, lui fait défaut. Rares sont les diaristes qui ferment leur cahier, et s'en repartent, comme Roquentin, la conscience relativement tranquille de qui a plus ou moins bien accompli sa tâche. Le journal ne double l'enquête policière que dans la fiction. Et il n'est pas davantage une version intimiste du roman d'aventure. Son territoire est semé d'embûches, ses indices évanescents. Quel que soit le filet - et peu importe si les mailles en sont plus ou moins serrées - qu'il jette sur la réalité, quelque chose s'en échappe qui lui interdit de la comprendre dans son ensemble. Il lui manque toujours la pièce décisive, celle qui le hante comme elle hante la conscience de son auteur:

C'est cet "objet", cette chose extérieure, tendre et introuvable qu'il faudrait définir. Et définir, ce serait - bien entendu - la trouver. Toute ma vie se passe dans cet état d'angoisse et d'oisiveté, de désir qui ne dépasse jamais la rêvasserie. Toute ma peur de la mort est liée au désir angoissé de cet objet impossible à découvrir (18 mai 1939, p. 324).

Mais alors pourquoi tant d'obstination? Où, et à travers quel mécanisme de compensation, le journal trouve-t-il la force de surmonter les frustrations qui scandent sa rédaction, de contrôler ce que parfois il faut bien appeler son caractère compulsif?

Comme le phénix qui renaît de ses cendres, le journal s'alimente de sa perte, là où il se perd, dans le quotidien, dans la puissance diabolique du quotidien:

Quels que soient ses aspects, le quotidien a ce trait essentiel: il ne se laisse pas saisir. Il échappe. Il appartient à l'insignifiance, et l'insiginifiant est sans vérité, sans réalité, sans secret, mais est peut-être aussi le lieu de toute signifiance possible. ${ }^{5}$

Diabolique parce qu'ainsi que le remarque Blanchot, la banalité et la platitude du quotidien pourraient être aussi - c'est une hypothèse condamnée à ne connaître ni démenti ni confirmation - le plus important. La profondeur et le tragique seraient alors - et à ce point le jeu ne vaut-il pas la chandelle? - l'autre

5 Maurice Blanchot, "La parole quotidienne", L'entretien infini, Paris, Gallimard, 1969, p. 357. 
face du superficiel et de la nullité. Les paris restant ouverts, chacun est libre ou non de miser. De se lancer dans un jeu dont la seule règle reconnue de tous est que, dans son alternance de temps forts et de temps faibles, il ne connaît pas de fin. Comme le mouvement de ce pendule dont Leiris, qui a joué jusqu'au bout, a fait son emblème: "un pendule oscillant entre un point d'exclamation et un point d'interrogation" (9 décembre 1981, p. 741).

\section{Une occasion perdue}

\section{Apprendre à dire Je}

C'est à l'adolescence - "vers l'âge de quatorze ans" - que Leiris fait remonter, dans L'âge d'bomme, l'habitude d'inscrire "poèmes et pensées" (p. 162) dans un cahier alors à tranche rouge et couverture de toile grise. Dans Biffures, il rattachera à l'enfance celle de "me faire des albums ou des cahiers, de réunir en une quantité limitée de feuillets ou sous un nombre réduit de couvertures (à moins que ce ne fût dans des tiroirs ou dans des boîtes) ce qui pouvait me plaire ou m'intéresser [...]" (p. 276) ${ }^{6}$. Double et lointaine origine dans laquelle se défait la notion même de commencement et, avec elle, celle de fin. Mais aussi, et tout aussi bien, celle d'inachèvement.

Où et comment pourrait s'achever ce qui n'a pas commencé? Il y a certes dans le Journal - tel qu'on peut le lire aujourd'hui dans la remarquable édition de Jean Jamin - une première et une dernière phrases mais celles-ci ne sauraient être investies d'un sens particulier. Elles marquent simplement les termes $a b$ quo et ad quem de l'axe chronologique sur lequel se greffent les entrées, selon un rythme que celui-ci ne commande guère. Il n'est probablement pas indifférent, à ce propos, de remarquer que les premières pages ont été arrachées et en outre que le déchiffrement des derniers mots reste incertain: "Vouloir interrompre [deux mots illisibles], pas [très] prudemment, ne vient que de moi" (7 novembre 1989, p. 809). Non pas pour dénoncer la 
présence d'une lacune mais plutôt pour renforcer ce trait caractéristique de tout journal, à savoir qu'il peut être interrompu à tout moment, par la mort, bien sûr, mais aussi par n'importe quel type de décision textuelle ou extratextuelle.

Mais revenons au commencement qui apparaît rétrospectivement comme le résultat d'une suite d'opérations de caractère propitiatoire qui repoussent aussi longtemps que possible l'inscription de la date inaugurale: Vendredi 27 octobre 1922 . Il y a d'abord la destruction matérielle des pages initiales. Puis, dans ce qui résulte, à ce point, le premier feuillet, deux anomalies. D'une part, le caviardage pointilleux des premières lignes (que contenaient-elles de si répréhensible?). De l'autre, et à l'opposé, un incipit dont le contenu apparaît énigmatique à moins d'en faire un subtil mécanisme de captatio benevolentioe qui, dans la communauté du "nous", offrirait le modèle d'une expérience fusionnelle qui pourrait être aussi celle de la lecture à venir:

[...] Peu à peu, par suggestion, nous devenons l'animal, et mentalement nous remuons, nous nous endormons ou nous faisons l'amour $[\ldots]$. Nous respirons difficilement, forcés que nous sommes de suivre le rythme haletant de l'orchestre. [...]. Et quand l'orchestre s'arrête, nous sommes étonnés de ne pas mourir par la cessation brusque de toutes nos fonctions ([Sans date], p. 27).

La description détaillée mais privée de toute forme de détermination spatio-temporelle de l'impact de la musique de Stravinski se présente alors comme une forme de précaution mais aussi d'invitation à aller de l'avant, après quoi le départ peut-être donné:

Vendredi 27 octobre

Dîner chez André Masson.

André Masson est avec Picasso le plus grand peintre actuellement vivant [...]. Son accueil est toujours cordial, dans le petit atelier qu'il occupe avec sa femme, sa petite fille et son ami Roland Tual à qui je dois d'avoir fait sa connaissance. Cette après-dînée, nous rîmes comme des fous (p. 26-27).

Phrase nominale, usage de la première personne pronominale singulière et des déictiques, datation: le dispositif minimum de l'écriture diariste est en place. Le Joumal accepte finalement, semble-t-il, de se reconnaître et de se donner à lire comme tel. 
Et peu importe à ce niveau de l'interprétation que la rédaction ait commencé ou non dans la clandestinité domestique. D'une part parce que Leiris, qui en maitrisait parfaitement les mécanismes, eut tout loisir, quand le moment fut venu, de modifier le protocole de la destination inhérent au journal comme à toute forme d'écriture. De l'autre, parce que Zette d'abord, le lecteur ensuite ont vite pris - avec les conséquences que cela imposait - la place de 1'، interlocuteur fictif (partenaire presque imaginaire)" (Biffures, p. 184) auquel le texte s'adressait initialement ${ }^{7}$ :

Je sais maintenant (et c'est à peu près entendu entre nous), que ce cahier lui [Zette] est destiné, comme une sorte de testament. Que va-t-il en résulter quant à sa rédaction (14 juillet 1940, p. 329)?

À l'automne 1922, la question de la destination ne se pose pas mais plutôt celle de l'appropriation de la première personne pronominale. L'emploi inclusif $\mathrm{du}$ "nous" postule certes l'existence d'un "tu" mais plus encore celle d'un "je" dont la réalité est loin d'être assurée. Il apparaît clairement que c'est dans la relation établie avec le destinataire textuel que Leiris trouve initialement le principe d'indentité minimum qui légitime sa prise de parole. Au cours des deux premières années, en tant que sujet de l'écriture, il semble obéir à une urgence prioritaire: articuler, le plus souvent hors du temps et des personnes - jamais comme dans ces premières pages il n'aura posé autant d'absolus, proféré autant de vérités - une série d'énoncés dont la modalité assertive tend, avant toute chose, à renforcer l'espace de l'énonciation, à en assurer le prolongement:

La poésie n'est pas définissable, car elle est partout. Elle est partout car elle est en nous (14 décembre 1922, p. 28).

Le monde est une armoire à glace dont nous n'avons pas la clef: nous nous voyons dans le miroir sans savoir ce qu'il y a derrière (25 février 1923, p. 32).

7 "[...] ce journal, en principe non destiné à la publication, mais dont je compte pourtant qu'il paraîtra post mortem (30-10-69)" (note au 20 avril 1968, p. 626). Dès Biffures la question de la publication semble en fait être réglée. Décédée avant Leiris, Zette ne lut jamais le Journal. Seule Léna, protagoniste de l'année 1934, semble l'avoir lu de son vivant: "Léna et sa curiosité: alors qu'elle me connaissait à peine elle a lu mon journal " 21 mars 1934 , p. 256). Sur le rapport lecteur-destinataire (et dans le prolongement de J. Rousset, "Le journal intime texte sans destinataire", dans Le lecteur intime, Paris, Corti, 1986, M. Calle-Gruber, "Journal intime et destinataire textuel", Poétique, no 59 , septembre 1984, p. 390-391. 
Beaucoup plus problématiques apparaissent les rares emplois de la première personne à laquelle fait défaut l'unicité qui aurait dû fonder le sentiment de son identité. S'il est vrai que "je ne peut être identifié que par l'instance de discours qui le contient et par là seulement " 8 , à qui rapporter les fragments narratifs ou les descriptions circonstancielles qui mettent en jeu un sujet qui évolue plus volontiers dans le monde du rêve ("Cette nuit j'ai rêvé que je me promenais le long d'une vaste avenue qui longeait un grand bâtiment sombre, un hospice d'aliénés [...] ", 12 avril 1923, p. 32) ou de la surréalité ("Vers cinq heures du soir, place de la Madeleine, j'ai failli m'étouffer en avalant un iceberg ", 31 juillet 1923 , p. 33) que dans celui de la veille, totalement oblitéré dans ces premiers feuillets? Leiris éluda la question aussi longtemps qu'il ne fut pas en mesure d'assumer plus ou moins explicitement l'ensemble de son histoire, celle qui, pour relever aussi du temps et de l'espace réels, ne pouvait ignorer "la banalité de la vie".

Cet instant on peut le situer sans difficulté au printemps 1924. Après avoir reconduit l'énoncé à l'ici et maintenant de l'énonciation ("Révision des épreuves des Pincegrain chez Marcel Jouhandeau. Je lis Kraquelin. / Dîner avec Jouhandeau et André Masson [...]" 13 mars 1924, p. 34), l'écriture met alors en scène pour la première fois le corps et ses désirs. C'est la relation d'une "aventure pédérastique" (probablement avec Jouhandeau, présence obsédante de cette première partie de l'année) dont le récit, plus tard censuré 9 , semble avoir provoqué un véritable traumatisme dans le texte contraint, à ce point, à voir la réalité la sienne comme celle qu'il avait charge de transcrire - "Sans masques [...ni] lunettes déformantes". Trois mois plus tard, assurée d'une assise plus large, la scène de l'écriture se retourne pour la première fois vers ce qu'elle sait ne plus être et tire de cette différence la force de se définir:

8 Émile Benveniste, Problèmes de linguistique générale, Paris, Gallimard, "Bibliothèque des sciences humaines", 1966, p. 252.

9 On en retrouve la trace dans un feuillet non daté, collé postérieurement - à la date de sa rédaction (automne 1929). Il s'agit d'une "Note remise au Dr. Borel, au début de l'analyse" et au terme de laquelle on peut lire: "La gêne dont je me plains est apparue sous forme de gêne physique (douleur et contraction anormale d'un des testicules) après une aventure d'ordre pédérastique relatée dans le cahier jaune (fin mars - début avril 1924)" (p. 204). Le récit en avait été en toute probabilité transcrit sur la page arrachée à la fin de l'entrée du 30 mars 1924 ( $c f$. note 8, p. 836). 
Coup d'œil rétrospectif sur ce journal [...] (9 juillet 1924, p. 48).

À preuve de ce nouveau statut ${ }^{10}$, et dans la foulée d'un impitoyable "bilan sentimental", est insérée "une confession" écrite quelques mois plus tôt — "[...] Ma vie est plate, plate, plate [...]" - mais alors exclue du corps du texte, probablement pour sa trop forte teneur émotive. D'un moindre risque - son caractère fantasmatique le dégageait de toute forme de responsabilité était par contre apparue, à peu près à la même époque, la transcription, elle-aussi rétrospective et "à titre documentaire", du bref récit en Je intitulé pour l'occasion, et afin d'éviter toute assimilation abusive, Le lycanthrope.

Du rêve au fantasme, du fantasme à la confession: on retrouve, dans l'acheminement du texte vers la prise en charge de son statut, certaines des étapes qui conduisirent Leiris à stipuler, en 1939 , le pacte autobiographique ${ }^{11}$. Mais alors qu'au moment où il publia L'âge d'bomme, l'attention de l'écrivain était concentrée sur la dimension contractuelle du pacte et ses implications ce qu'il appela la corne de taureau - elle s'arrête ici à l'étape préliminaire, à la reconnaissance de la triple équation auteur $=$ narrateur $=$ personnage:

[...] Je confesse mon apaisement. Je crois maintenant être capable de me distraire. [...] L'univers me sollicite de tous côtés: je puis voyager, avoir des aventures amoureuses, boire de l'alcool dans un autre but que celui de m'étourdir; - je puis à présent m'intéresser, et ne suis plus obligé de me "toucher pour savoir si je suis vivant". Un réveil. Je vis au participe présent (31 août 1924, p. 64).

Le plus difficile était fait. Il restait - ultime condition - l'acceptation d'un nom propre dont l'incertaine réalité agite ces premières années. Il faut attendre septembre 1926 pour que Leiris admette qu'il est bien celui qui dit "Je". Et encore le fait-t-il sous la forme parodique du travestissement, dans le prolongement des

10 C'est à travers l'emploi du déictique "ici " qu'en mars Leiris avait fait, pour la première fois, allusion au lieu d'inscription de son activité: "Je recopie ici, à titre documentaire, une prose (inachevée) que j'écrivis l'été dernier. Je l'intitule aujourd'hui Le lycanthrope [...], (30 mars 1924, p. 36).

11 Dans l'étude "Michel Leiris: autobiographie et poésie. (Le pacte autobiographique, Paris, Seuil, 1975), Philippe Lejeune a reconstitué les étapes de ce parcours avec une précision à laquelle on ne saurait apporter aucune modification. 
gloses du Forçat vertigineux, autre version fantasmatique du récit autobiographique ${ }^{12}$ :

Michel Leiris - Il me suffit de retourner mon nom (- Siriel Lechim) pour être instantanément transformé en un vieux rabbin (p. 125).

À ce point, même si les énoncés à la première personne restent rares, si le rêve plus que la veille continue à stimuler le procès d'identification, le journal a définitivement reconnu la légitimité de sa pratique. Elle ne fut jamais remise en question. Il y eut certes des vides, des suspensions mais à aucun moment d'interruption fût-elle matérielle. Pas de blancs ni de pages non écrites dans les cinq cahiers format écolier qui, sans solution de continuité, servirent de support à la rédaction. Comme si le noir de l'écriture avait pu en quelque sorte exorciser la discontinuité, boucher les trous du vécu. On retrouve ici l'image des polders, la nécessité d'opposer une résistance - la plus dérisoire fût-elle - "à la chose sans nom qui est en nous et dont le flux nous menace ", évoquée dans Fourbis (p. 26). Et cela jusqu'à l'inscription, au milieu du dernier cahier, de l'ultime entree suspendue au bord du vide dans lequel le silence puis la mort auront précipité l'écrivain.

Soixante-sept années, 5 cahiers, un total de près de sept-cent cinquante pages manuscrites. En aucune façon un résumé de l'œuvre ni même de la vie de son auteur. Si l'on veut, un "document" mais à condition de prendre le terme au sens actif qu'entendait Bataille lorqu'il en fit - au pluriel et en en privilégieant l'aspect opératoire - le titre de l'une des plus virulentes revues de l'entre-deux-guerres. Lisant le Journal, on sera dès lors moins sensible à sa dimension testimoniale (à ce qui le rattache au passé) qu'aux percées qu'il peut ménager, au renouvellement qu'il peut apporter dans la perception de l'ouvre et de la vie. On interrogera ses silences, on approfondira ses doutes. On réintroduira le jeu que la publication en volume a, par la force des choses, tendance à resserrer. On n'oubliera surtout à aucun instant que si les feuillets dont il se compose apparaissent "suppressiblels] à l'infini "13, c'est aussi parce que d'une instance à l'autre il n'existe aucune forme de hiérarchie, que, pour relever du

12 Version à laquelle il convient d'ajouter l'autobiographie de Michel Siriel dans Aurora.

13 Cf. les réflexions de Roland Barthes sur ce qu'aurait pu être son journal ("Délibération" (1979)), in Le bruissement de la langue, Paris, Seuil, 1984, 
quotidien, tout y est à la fois important et dérisoire. Que moins qu'ailleurs l'on ne saurait a priori y opérer de choix, privilégier un aspect plutôt qu'un autre. Ce serait trahir sa spécificité, se laisser prendre au mirage de sa saisie.

Le Joumal semble livrer plus facilement que d'autres formes d'écriture le secret de son fonctionnement pour la simple raison qu'il apparaît à chaque instant en train de se faire. Or, et quoi qu'il en laisse accroire - c'est un secret de Polichinelle -, il avance à tâtons vers un centre absent dans lequel se fourvoie toute forme de projet. Loin d'obéir à une stratégie d'ensemble, les entrées qui le composent sont autant de réponses ponctuelles au défi qui chaque jour se présente sous une forme nouvelle. Indépendamment du problème sémantique qu'elle assume en propre, chaque partie vaut ici pour le tout, si tant est qu'en l'absence d'orientation et de fin l'on puisse parler de tout. Ce qui ne veut pas dire qu'hétérogène et discontinu, le Journal se présente comme un ensemble indéchiffrable. S'il ne sait pas de quoi sera fait son lendemain, il ne continue pas moins à chercher un sens et une unité à chacune des étapes de son développement.

Ce n'est en effet que dans la perspective globale d'une interrogation indéfiniment reformulée que l'on ne saurait en privilégier un aspect plutôt qu'un autre sans altérer sa signification la plus profonde. Il en va différemment dès que l'on restreint la perspective à la façon dont, jour après jour, l'écrivain s'efforce ou non de répondre à la question qui le harcèle. Dans le parcours labyrinthique qui le conduit à la mort sans avoir jamais répondu, se perçoivent alors un certain nombre de directions qui plus que d'autres semblent avoir alimenté l'illusion de la réponse. D'autres qui se sont aussitôt révélées des impasses. Ce qui, vu de loin, apparaissait dépourvu d'éléments caractéristiques, insaisissable, prend peu à peu la forme d'un paysage dont les différents aspects stimulent, quant à eux, la saisie et l'analyse. Le sens qui jusque-là faisait défaut déborde de toutes parts. Exercice introspectif et cathartique, aide-mémoire, atelier d'écriture, recueil de rêves et de pensées, témoignage... Dans la multiplication de ses fonctions, le Journal, pour un peu, deviendrait l'œuvre totale en marge de laquelle se seraient écrites toutes les autres:

p. 410) et le commentaire de Gérard Genette ("Le journal, l'antijournal", Poétique, septembre 1981, no 47, p. 315-322). 
Un livre qui ne serait ni journal intime ni ceuvre en forme, ni récit autobiographique ni œuvre d'imagination, ni prose ni poésie, mais tout cela à la fois. Livre conçu de manière à pouvoir constituer un tout autonome à quelque moment que (par la mort, s'entend) il soit interrompu. Livre, donc, délibérément établi comme œuvre éventuellement posthume et perpétuel work in progress (26 septembre 1966, p. 614).

En fait, il n'en est rien. Qu'on le veuille ou non, le Journal, celui-ci comme un autre, est une donnée brute - sauvage - qui ne se laisse saisir qu'artificiellement. À travers un découpage arbitraire qui en altère le sens au moment même où il le multiplie. Pot-pourri domestique d'éléments résiduels, il relève d'une économie de la perte quelle que soit l'opération de recyclage dont il lui arrive parfois d'être l'objet. Leiris, quant à lui, savait que ses cahiers seraient un jour publiés. Mais il ne les écrivit à aucun moment dans cette perspective. C'était, compte-tenu de sa relative notoriété et de la demande éditoriale, une hypothèse plus que probable qui intègra et alimenta le procès de l'écriture quand son statut d'auteur ne fit plus de doute. L'ignorer aurait été faire preuve de mauvaise foi. Le reconnaître ne changeait rien quant à l'essentiel qui n'était pas de dire un peu plus ou un peu moins, mais, plus radicalement, de continuer à dire. Car - Barthes l'avait parfaitement formulé - "ce que le journal pose, ce n'est pas la question du Fou: "Qui suis-je?" mais la question comique, la question de l'Ahuri: "Suis-je?" "14.

Au cours de l'analyse qui va suivre nous nous efforcerons de ne pas oublier que chacune des entrées qui composent le Journal fut avant toute chose une façon de répondre positivement à cette question, de parer momentanément au défaut d'existence qui la formulait. Et cela quel que fût son contenu - faits et actes, idées apparues, sentiments éprouvés pour reprendre la distinction opérée par Amiel dans ce qui, selon lui, devait former ou composer la matière du journal.

\section{Où et comment dire Je?}

De la nécessité d'une telle composition, Leiris reconnaît la validité au moment où, conscient de sa déficience, il ébauche, 
38

pour la première et la dernière fois, ce qui aurait pu être (si elle avait été appliquée) une poétique du texte:

Le journal intime que je rédige en ce moment reste encore beaucoup plus un "cahier de notes" qu'un journal intime à proprement parler. Toutes mes réflexions sont bien trop théoriques; elles visent trop au général, tendent trop vers l'aphorisme. Je parle beaucoup moins de moi, que de mes opinions sur moi-même ou sur d'autres sujets. Pourtant, ce n'est pas de cela qu'il devrait s'agir, mais plutôt de projeter sur ces pages mon reflet, d'une manière absolument concrète ( 17 mai 1929, p. 169).

D'une extrême lucidité, cette réflexion intervient à quelques jours d'une ferme reprise en main du Joumal qui entend, plus ou moins explicitement, remédier à un regrettable mais incontestable état de fait - "Relisant ce cahier, j'observe combien d'année en année, les notes se raréfient. (Mai 1929, p.135). Au laconisme des rares entrées de 1926, 1927 et 1928, s'oppose à partir de cet instant une intense activité de l'écriture sur laquelle pèse un regard attentif qui la juge à plusieurs reprises avant de tirer les conclusions à peine citées. Il apparaît ainsi que le journal "peut mettre sur le chemin de la découverte" d'une "technique poétique" (p. 138); qu'il constitue "une sorte de rappel à la réalité, et un nouvel avertissement quant à la méfiance qu'il convient d'avoir à l'égard du verbalisme" (p. 142) et de la littérature (p. 152); qu'il est le lieu d'une autocritique, terme préférable à celui d'introspection "parce qu'il comporte quelque chose d'actif, alors que l'introspection se réduit à une contemplation purement passive de soi-même, pleine d'une détestable complaisance" (p. 144); qu'à condition de "ne déchirer aucune page" (p. 152), il peut servir à "boucher des portes, en y inscrivant tout ce qu'il ne faut pas faire" (p. 149). Ainsi de suite, jusqu'à la constation: "Je me lasse de ce journal ", après quoi Leiris affronte le problème de front et ébauche, au conditionnel, un manifeste dont les deux pivots seraient, portées à leur extrême, la sincérité et son corollaire immédiat, la transcription indifférenciée du réel:

Je voudrais tomber malade à force de sincérité. Donner l'exemple unique d'un homme qui, somme toute, s'est rarement illusionné sur lui-même et a su mieux que quiconque voir clair en lui. [...]

J'aimerais avoir le courage d'écrire dans ce cahier des choses de ce genre: aujourd'hui j'ai chié de telle manière, j'ai fait l'amour 
de telle autre, j'ai pensé cela de tel ou telle, je me suis branlé [...] (17 mai 1929, p. 168).

Derrière l'ombre tutélaire de Baudelaire et de Rousseau, le lecteur aura reconnu les arguments à travers lesquels Leiris s'efforcera en 1933 de défendre la matrice diariste de L'Afrique fantôme. Mais ici il ne s'agit pas tant de défendre une pratique que de l'instaurer. Et, avant même de l'instaurer, de lui trouver un cadre générique et des exemples, fussent-ils des contre-exemples! On remarquera avec quelle habileté, au moment même où il est contraint d'introduire pour la première fois le terme de ajournal intime " dont il a conscience qu'il pourrait mieux que tout autre définir ce qu'il voudrait (ou devrait) faire, l'écrivain trouve le moyen de se soustraire à son injonction en reconnaissant que seul "un fou ou réputé tel" - en l'occurence Louis II de Bavière - y a jusqu'ici répondu.

Ce sera un véritable échec. Quels que soient l'application et le volontarisme avec lesquels, folie ou pas, l'écrivain se met à la tâche, sa tentative n'aboutit pas ou, plutôt, pas là où elle aurait dû le conduire. Il ne lui faut pas plus d'une quinzaine de jours pour reconnaitre qu'"on ne trouve dans ce cahier aucune de ces phrases capables d'enflammer le papier, comme dit à peu près Baudelaire dans la préface de Mon cour mis à nu" (4 juin 1929, p. 188). Et cela malgré le tour nouveau qu'a pris momentanément la rédaction qui n'apparaît plus trouée par les silences et les vides qui pouvaient jusque-là entraver la lecture mais au contraire appesantie par un excès d'information qui, en fin de compte, ne fait pas avancer grand chose quant à la solution du problème. Paradoxalement, c'est au moment où l'écrivain accepte d'assumer la tâche - favoriser l'émergence de l'intimité - qu'il reconnaît alors être prioritairement la sienne, que vient à manquer ce qui devait en constituer la fin - la sincérité.

Mais Leiris fut-il jamais convaincu qu'il lui aurait suffit de renoncer à l'abus qu'il avait fait jusque-là du présent intemporel, de se soumettre aux urgences de l'heure et de l'instant pour atteindre cette dimension du sujet dont il percevait qu'elle faisait défaut à son cahier? N'y a-t-il pas dans la rapidité avec laquelle il modifie radicalement l'objet et les modalités de l'énonciation de quoi éveiller le soupçon?

J'ai rempli mon stylographe d'encre fraîche [...]./ Je suis vêtu d'un veston d'intérieur bleu vif, à boutons dorés, genre blazer. 
40

Sur ma table il y a cigarettes, buvard, crayons, livres, papier, etc. Je suis assis face à la fenêtre et j'aperçois le temps qui se couvre légèrement et le soleil qui descend. J'entends le tic-tac de ma petite pendule, la porte des cabinets qui se referme sur une des domestiques qui vient d'y entrer, de vagues bruits d'avion et de tramways. Maintenant c'est la chasse d'eau qui se fait entendre [...]. Tout cela est naturellement sans intérêt, et je dois bien convenir que j'ai une terrifiante quantité de temps à perdre, pour m'amuser ou m'astreindre à noter de pareilles futilités (17 mai 1929, p. 170).

Qu'il en serait allé ainsi, Leiris l'avait pressenti alors même qu'il acceptait de se plier à une intimation dont force lui était de reconnaître l'inéluctabilité: "Mais je suis bien obligé de me dire que relater est peut-être nécessairement égal à frelater" (p. 169). On retrouve dans cette nouvelle modalisation de l'énoncé une ultérieure manifestion de la conduite de protection à l'abri de laquelle l'écrivain s'est aventuré là où, à ce point, il ne pouvait plus ne pas se diriger. Un peu comme s'il s'était dit: si journal intime il doit y avoir, autant y aller carrément en jouant cartes sur table. Après quoi, on - mais qui ? - n'aurait eu plus rien à lui reprocher. Et surtout pas l'insignifiance dans laquelle il eut très vite la sensation de s'enliser:

Je suis obligé de constater que jusqu'à présent ce journal est très pauvre et ne m'apprend rien sur moi. Tout au plus, il me permet d'avoir l'illusion que je fais mieux que rien, - ce qui serait encore à discuter ( 28 mai 1929, p. 187).

Que d'obligations là où, en l'absence d'ordre et de hiérarchie, aurait dû régner une totale liberté. Et puis la pauvreté, la facilité, la mesquinerie dont, tour à tour et chaque fois qu'il se relit, l'écrivain se reconnaît coupable ne sont-elles pas autant de prédicats littéralement privés de sens dans l'espace qui est censé être celui de l'épiphanie de la sincérité? En fait, tout au long de ces pages artificielles comme peut l'être tout exercice d'entrấnement, Leiris se révèle incapable de contenir le "pouvoir de dissolution" inhérent au principe d'indifférenciation dont il venait de faire la règle d'or du journal intime. Trop lourd est le poids des mots, trop concrète l'image que véhiculent des instances qui ne portent ou tendent à ne "porter la trace d'aucun choix quant aux événements relatés. (p. 169) et trop fragile le cahier pour continuer à en être le support. Aussi plutôt que de risquer l'effondrement et l'abandon qui en aurait résulté, juge-t-il plus sage de déplacer le lieu de la collecte. Le 28 octobre, l'inscription 
"PYSCHANALYSE " ${ }^{15}$ suspend - ce sera la plus longue interruption de l'ensemble du texte - la transcription. Celle-ci reprendra le 21 avril 1933 mais à des conditions et sous des modalités qui n'auront plus rien à partager avec l'intense travail qui pendant quelques mois a coincidé - ne fussent que dans les intentions - avec ce qui aurait pu être le journal intime de l'écrivain.

D'aucuns s'étonneront de la relativement faible teneur introspective d'un journal dont on aurait pu croire - ne fut-il pas écrit par un spécialiste de la confession? - qu'il aurait été pétri d'intimité. L'Afrique fantôme n'accepta-t-elle pas de payer très cher son acharnement à capter le réel objectif et subjectif sous toutes ses formes, et cela en empruntant au journal la liberté d'une totale indifférence à un sens extra-textuel? Comment, à ce point, ne pas se demander pourquoi ce qui fut possible au cours de la Mission Dakar-Djibouti ne l'avait-il pas été quelques années plus tôt et, plus encore, quel élément a pu enrayer le mécanisme de transcription qui, somme toute, se remit en marche sans trop de difficulté peu de temps après le retour d'Afrique?

À lire le texte qui ne mentionne ni l'interruption (octobre 1929) ni la reprise (21 avril 1933) en tant que telles, Leiris semble être retourné à son cahier comme à la plus naturelle des destinations: dans le prolongement du manuscrit que Gallimard était en train de publier. Pendant près de trois ans, les entrées se suivent à un rythme relativement régulier, avec des hauts et des bas certes, mais sans mettre en cause le protocole du Joumal, tel qu'il avait été reformulé au cours d'une discussion littéraire avec Schaeffner, lors d'une halte dans le Cameroun du Nord. Sans prétendre à l'exhaustivité - il n'en a guère le temps - l'écrivain continue à être d' avis qu'il faut tout raconter", sans "choisir" ni "transfigurer" (L'Afrique fantôme, p. 151). Le futile comme le grave, ce qui peut paraitre superficiel comme ce qui met en cause les couches les plus profondes de l'être. Les dîners avec les Noailles et la longue relation avec Léna, objet en 1934 d'un Journal de ma vie officieuse. L'obsession de la mort, le poids de l'ennui, la menace de la guerre mais aussi les gestes qui scandent la vie quotidienne faire ou non l'amour, rencontrer des amis, rêver. Le problème

15 Il s'agit d'un lapsus relevé quelques années plus tard: "Lapsus remarqué seulement le 10 mars 1946: "pyschanalyse", comme si cela avait quelque chose à voir avec les poissons. La pêche dans l'eau trouble de l'inconscient." (p. 203). 
42

n'est plus de se reconnaitre et de s'accepter, de régler le principe osmotique entre les différentes instances du moi. Le cahier a perdu la précarité qui l'apparentait à un phénomène transitionnel. Quelle que soit sa fragilité, l'identité du sujet de l'écriture est désormais assurée. Quant à la sincérité dont le fantasme encombrait la rédaction, elle n'est plus liée au secret, à son dévoilement c'est-à-dire à l'objet plus ou moins compromettant de l'écriture les "phrases capables d'enflammer le papier" — mais à l'écriture elle-même. Ce qui déjà pointait en 1929 dans l'équation relater = frelâter mais n'avait pas alors été approfondi et pour cause. Il en va différemment maintenant que l'écrivain a non seulement reconnu la légitimité de "la littérature "de confession" " mais en a fait la seule forme d'écriture possible pour lui:

Pas d'autre forme littéraire actuellement possible - au moins pour moi - que la littérature "de confession". Ne pas écrire mes mémoires (ce qui serait pour le moment absurde) mais soit des tranches de vie (par exemple: toute ma vie sexuelle depuis mon enfance jusqu'à la perte de ma virginité avec D[aisy] S...), soit des sortes de corpus groupés en raison d'une identité de nature, soit des essais théoriques basés sur l'expérience personnelle (par exemple: essai sur la masturbation, — sur le déguisement) (24 août 1933, p. 230).

Un rien et Leiris aurait reconnu l'insuffisance du journal auquel n'est prêtée ici aucune qualité d'ordre littéraire. Et c'est bien sur ce terrain que va se jouer le sort d'une entreprise qui apparait peu à peu privée de ses attributs les plus significatifs. Car même si la formulation est encore vague, il est clair que c'est ailleurs que Leiris a décidé de "se confesser". Ailleurs - le projet a déjà forme et consistance - mais pas n'importe où. Il est en effet assez troublant de voir ébauchés, dès 1933, non seulement certains des thèmes de L'âge d'bomme mais aussi, et d'une manière encore plus significative, le principe génétique de La règle du jeu ainsi que la perspective phénoménologique du "Sacré dans la vie quotidienne. Deux jours plus tard, les intentions se précisent et Leiris commence la collecte des matériaux - "Rechercher les documents utilisables dans mon courrier. Consulter tous cahiers, notamment celui de poèmes" (26 août 1933, p. 231) - d'une autobiographie sentimentale" qui a pour titre possible: Jeunesse de Damoclès Siriel. De ce projet qui entre temps deviendra L'âge d'bomme, on ne retrouve plus mention jusqu'en octobre 1934. L'écrivain a interrompu sa relation avec Léna et depuis quelque mois il a repris la cure psychanalytique. Après avoir mentionné 
comme "Titres de livre: Haut mal (poèmes) / L'âge d'bomme", il ébauche en quelques lignes extrêmement précises le contenu et le sens de l'ouvrage qu'il ne lui reste plus désormais qu'à rédiger:

Mémoires. Retracer patiemment toute sa vie avec toute la précision désirable; revivre un à un chaque événement et tout fixer avec le maximum de détails, sans se préccuper d'être ou non fastidieux. Peindre sa vie comme un portrait de primitif, afin par la vertu de ce portrait - de donner une consistance à son être. Non pas ressasser le passé, par masochisme de mémoire, mais le ressusciter, le sculpter aussi ressemblant que possible, en faire une sorte de solide. Il ne peut être question de truquer, car se serait mourir que de truquer (12 novembre 1934, p. 288289). ${ }^{16}$

Le 26 décembre 1935, Malraux en avait lu le manuscrit. À ce point, et alors que la faible quantité des entrées laisse présumer que l'année 1935 a été occupée "littérairement" à la rédaction de ce premier essai autobiographique, le sort du Joumal était décidé. Mais encore fallait-il le reconnaître et lui assigner la place qui désormais serait la sienne, à la périphérie de l’œuvre, dans ses marges.

\section{L'échec du Journal}

Fort du texte qu'il vient d'achever et de ceux dont le projet ne fait plus de doute, Leiris affronte finalement le taureau par les cornes et place au centre de l'écriture, dans des termes tels que le journal ne pourra y satisfaire, la question de l'autre et de l'unité du moi. L'impact violent provoqué par la lecture de L'âge d'bomme sur ses amis les plus proches - "Je voudrais que mes amis se rendent bien compte que L'âge d'bomme est une liquidation" ( 7 janvier 1936, p. 298) - l'a poussé à sortir de ses retranchements et à mettre les choses au clair, ne serait-ce que pour lui ie "Prière d'insérer" qui accompagne la publication du volume en 1939 soutiendra publiquement ce qui est ici débattu:

16 On remarquera que c'est sur une feuille volante qu'a été rédigée, en avtil 1934, la première version du fameux incipit ("Je viens d'avoir trente-quatre ans, la moitié de la vie. Au physique, je suis de taille moyenne [...]", L'âge d'bomme, p. 26) et que celle-ci n'a été collée dans le cahier qu'à la date du 7 juin, précédée alors de la mention "Début d'autobiographie rédigé en avril 1934. (p. 277). À cette date, Leiris a reconnu la validité de la perspective rétrospective. 
Nul soulagement à tenir un journal, à rédiger une confession. Pour que la catharsis s'opère, il ne suffit pas de formuler, il faut que la formulation devienne chant. Chant $=$ point de tangence du subjectif et de l'objectif [...] (8 janvier 1936, p. 299).

Il ne saurait faire de doute en effet que la mise en cause du journal est le prix à payer à l'hypothèque qui pèse sur l'escrivaillerie coupable et dont il importe prioritairement à l'écrivain de libérer l'entreprise autobiographique. Or qu'est-ce qui distingue à ce niveau le journal de l'essai autobiographique si ce n'est la mise en forme qui permet au second de franchir l'espace domestique et de trouver une occasion de rachat à travers le contact et la reconnaissance de l'autre? Alors que pour assurer le prolongement de la rédaction de L'Afrique fantôme telle qu'il entendait alors la poursuivre, il avait dû défendre le postulat "c'est par la subjectivité (portée à son paroxysme) qu'on touche à l'objectivité", Leiris retrouve ici une préoccupation plus lointaine: celle qui l'avait empêché d'adhérer totalement à la poétique surréaliste. De façon inattendue réapparaît le "souci compositionnel" qui, dans son esprit comme dans celui de ses compagnons de la rue Blomet, devait assurer à leurs productions respectives "une cohérence comparable à celle d'un organisme vivant ou d'une architecture musicale" ${ }^{17}$. Expérience exemplaire limitée dans le temps et dans l'espace, la participation à la mission Dakar-Djibouti avait assuré au texte qui avait charge de la transcrire la légitimation et le cadre formel à l'intérieur duquel chaque énoncé avait trouvé sa place et sa raison d'être. Les formes du contenu - l'intimité débordante - et non celles de l'expression avaient été mises en question et encore ne l'avaient-elles été qu'au nom d'une idéologie dont l'apprenti-ethnologue ne partageait aucun des préjugés:

Il y a maintenant bientôt deux ans qu'a paru ce malheureux livre, et, dans le milieu des professeurs, on en parle toujours de la même façon. Mauss déclare que je suis un "littérateur ", que "je ne suis pas sérieux "; il répète encore que ce livre a été très nuisible pour les ethnographes auprès des coloniaux. Je suis assez flatté, au fond, de ce rôle de bouc émissaire, malgré les ennuis que cela peut mattirer. [...] Mes amis ethnographes (telle Denise Paulme) s'inquiètent déjà de mon prochain livre; il faut que je m'en enorgueillisse en me disant qu'il y a, certes, du mérite à vouloir dire la vérité... (3 avril 1936, p. 302-303).

17 Michel Leiris, "45, rue Blomet", Zébrage, Paris, Gallimard, coll. "Folio/ essais", 1992, p. 227-228. 
Il en va différemment dans la version domestique qui ne dispose d'aucune forme de légitimation (interne aussi bien qu'externe) quel qu'ait pu en être, nous l'avons vu, le désir du diariste. En fait c'est pour n'avoir jamais véritablement cru au bien-fondé de son projet que Leiris n'a pu le porter à terme. L'analyse aidant, il ne lui fallut probablement pas longtemps pour se rendre compte qu'on ne tombait pas malade de sincérité et que si sincérité ou courage il y avait c'était ailleurs que dans la multiplication d'énoncés plus ou moins scandaleux qu'il fallait les chercher.

Le problème étant mal posé, il fallait lui trouver une nouvelle formulation et sortir de l'impasse où risquait de conduire L'Afrique fantôme, expérience unique et qui telle devait demeurer. Car si, à sa défense, l'élève de Mauss avait pu s'en remettre à l'exemple de Montaigne et de Rousseau, le "littérateur ", quant à lui, ne pouvait continuer à faire appel à l'objectivité et au calcul de l'erreur comme à la panacée qui aurait soulevé les objections qui s'opposaient à l'actualisation du projet du 17 mai 1929. Il apparaissait chaque jour plus clairement que tant qu'il restait dans le régime autarcique du journal ${ }^{18}$, il lui était difficile de franchir la barrière qui le séparait de l'autre conçu non plus comme une entité abstraite - le "général " - mais comme le "tu" sans lequel ne saurait fonctionner le circuit de l'échange. "Chant", "communion", "communication" sont, à quelques mois de distance, les termes dans le défaut desquels s'inscrit la condamnation du Journal, telle qu'en avait été fantasmée, à l'origine, la fonction salvatrice. Pour qu'il y ait "catharsis", "liquidation", il ne suffisait pas de mettre à nu "certaines obsessions d'ordre sentimental ou sexuel ", encore fallait-il les "confesser publiquement" et selon un "rituel" parfaitement réglé.

Sur cette nécessité et les modalités qu'elle emprunta, Leiris s'expliqua publiquement à deux occasions: à la sortie de L'âge d'bomme en 1939 et, de façon plus détaillée, en 1946, lors de la réédition de l'ouvrage, dans la préface "De la littérature considérée comme une tauromachie». En 1936, alors que n'avait pas

18 Préoccupation qui n'effleura pas un instant la rédaction de L'Afrique fantôme que celle-ci ait été ou non destinée à la publication. En s'adressant initiale. ment à la communauté scientifique, Leiris tendit à garantir l'aspect "documentaire" de l'ouvrage, à renforcer sa qualité d'objet en face du sujet qui l'aurait ou non étudié. En aucune façon à établir un circuit d'échange entre objet et sujet. 
46

encore mûri la décision de la publication, il n'en avait pas moins trouvé ce qui restera jusqu'à la fin le mot clef de sa relation à l'autre, le garant de son authenticité - "cette résonance si difficile à définir ":

Faire chanter les mots, comme un peintre peut faire chanter ses couleurs: chercher à être entendu de partenaires privilégiés qui vibreront à l'unisson ou plutôt de ce qui, chez ses partenaires, est susceptible de vibrer avec vous ( $\dot{A}$ cor et $\grave{a} c r i, \mathrm{p} .103$ ).

Il appartiendra à la métaphore tauromachique de dévoiler le travail extrêmement précis et pointilleux qui de la simple parole conduit jusqu'au chant, hypothétique point de tangence du subjectif et de l'objectif. De préciser qu'à l'"aspect tactique " - ne pas être submergé par le flot des mots, en faciliter l'affleurement à la surface de la page - s'ajoute un "aspect esthétique" — opérer la transmutation du discontinu et de l'hétérogène, le passage du subjectif à l'objectif :

Cette "formulation" ou mise en forme est ce qui objective (pose hors de moi, érige en objet valable pour les autres) les sentiments, représentations, pensées, etc., qui jusque-là n'étaient que subjectifs, donc prisonniers du cercle de ma mort. Impossibilité de se passer de ce côté formel, faute de quoi le passage du subjectif à l'objectif ne se produit pas ( $1^{\text {er }}$ octobre 1941, p. 342).

De même que pour ceux qui en ont fixé le cérémonial, les règles de la corrida devaient empêcher que le combat dégénérât en une boucherie, celles que se donna l'écrivain tendaient essentiellement à éloigner le risque de logorrhée, d'enfermement qui menaçaient sa pratique de diariste. Car il fut clair que si journal et autobiographie affrontent une matière identique, ils le faisaient selon des protocoles qui les exposaient à des risques différents.

Tout bien pesé - le renvoi de la parution de L'âge d'bomme donne la mesure de son hésitation - Leiris opta pour le risque autobiographique. Le 30 octobre 1936, le journal enregistrait sa défaite:

Absurdité fondamentale du "journal intime" qui constitue presque une contradiction dans les termes: non seulement je formule pour moi et je ne parle qu'à moi-même (faisant ainsi du langage - chose sociale - un usage strictement individuel [...], mais je m'échelonne le long des dates, choses extérieures à moi qui constituent mes repères essentiels; je me pense par jours, par mois, par années; je désagrège donc mon intimité pour la soumettre aux cadres les plus impersonnels, à ceux qui, 
par définition, lui sont le plus étrangers (30 octobre 1936, p. 306).

Frappée d'absurdité, son écriture relevait du défi, de la gageure perdus d'avance. Faible intérêt certes mais qui lui assurait une survie dont l'économie de l'œuvre et de la vie - Leiris en fut toujours parfaitement conscient - ne pouvait se passer. Les moments viendraient où seule la page du cahier serait en mesure de contenir la fragmentation, la dispersion dont désormais l'autobiographie avait charge - mais serait-elle toujours en mesure de l'assumer? - de combler les vides et les lacunes, de les représenter. C'est pour être sûr qu'en ces moments de désarroi où les mots comme l'identité viendraient à manquer - "Aussi seul que si j'étais abandonné; aussi dénué qu'un pauvre; aussi perdu qu'un chien. D'où le refuge de ce journal" (18 janvier 1934, p. 250) il aurait un espace où laisser une trace que l'écrivain n'abandonna pas ses cahiers, quitte, entre temps, à les reléguer à des occupations subalternes ${ }^{19}$.

Mais là encore le Journal dut mesurer ses limites. La page se révéla parfois trop lisse, trop fragile pour soutenir le poids des mots, servir de support au travail de l'identité préliminaire à toute prise de parole:

Écrire me délivre de l'angoisse, mais il arrive que mon angoisse soit si grande qu'il m'est alors impossible d'écrire. Il y a en ce cas cercle vicieux et le remède me fait défaut quand jen aurais le plus besoin (12 novembre 1980, p. 732-733) ${ }^{20}$

Ce sont les longues années de silence, celles où le rythme de la transcription ralentit à la limite de la paralysie, qui précèdent

19 Sur la fonction de relai de l'écriture domestique, $c f$. également: "Régulièrement, mon angoisse m'amène à penser au suicide; régulièrement je ne fais pas un pas. Il se peut également que me prenne alors le désir d'écrire quelque chose - quelque chose de littéraire et de "transmué", s'entend. Presque toujours je n'écris rien, ou bien il arrive que je me replie sur ce cahier et que j'y inscrive quelque chose - histoire d'écrire, ou plutôt: comme si je faisais mes comptes [...]" (18 mai 1939, p. 323).

20 Très vite, Leiris établit la relation dépression-écriture qui caractérise pour ainsi dire tous les journaux intimes: "Écrire ne m'arrache plus, comme naguère, à l'angoisse... Peut-être y a-t-il un cercle viceux? Angoissé de plus en plus, j'écris de plus en plus mal de sorte que, moins que jamais, cette écriture n'a le pouvoir de contrebattre l'angoisse. Et cela justement quand, l'angoisse, étant accrue, il faudrait que cette écriture soit plus puissante que jamais!: (17 novembre 1978, p. 693). 
48

l'explosion des crises les plus graves: l'abandon du surréalisme et le début de la cure psychanalytique en 1929, le départ pour l'Afrique en 1931, la tentative de suicide en 1957. Les blancs qu'elles inscrivent dans le déroulement de la rédaction sont aussi éloquents que l'intense activité introspective qui leur fait suite, à peine la crise a-t-elle trouvé, quelle qu'elle soit, une issue. Ainsi, symboliquement reliée par la post-datation au geste même du suicide et matérialisée par l'ouverture d'un nouveau cahier, la reprise de l'écriture qui interrompt le quasi-silence du début des années cinquante ${ }^{21}$ :

Nuit du mercredi 29 au jeudi 30 mai

Ingestion, après boire, de 5 à $6 \mathrm{~g}$ de phénobarbital, ce qui nécessite mon transfert à l'Hôtel-Dieu puis à l'hôpital ClaudeBernard. Je commencerai à reprendre un peu conscience le dimanche soir et c'est à l'hôpital même que je commencerai, d'abord de façon tout à fait informe et décousue, la rédaction d'un nouveau journal, sur un cahier scolaire qui porte la mention "Lutèce" au-dessus d'une vignette en couleurs représentant le square du Vert-Galant (p. 494).

21 On ne compte que trente entrées diluées sur dix-huit pages de 1950 à 1956. 